IJMMS 28:11 (2001) 673-678

PII. S0161171201011292

http://ijmms.hindawi.com

(c) Hindawi Publishing Corp.

\title{
ON THE CONSTRUCTIONS OF TITS AND FAULKNER: AN ISOMORPHISM THEOREM
}

\author{
SUDHIR R. NATH
}

(Received 19 December 2000)

\begin{abstract}
Classification theory guarantees the existence of an isomorphism between any two $E_{8}$ 's, at least over an algebraically closed field of characteristic 0 . The purpose of this paper is to construct for any Jordan algebra $J$ of degree 3 over a field $\Phi$ of characteristic $\neq 2,3$ an explicit isomorphism between the algebra obtained from $J$ by Faulkner's construction and the algebra obtained from the split octonions and $J$ by Tits construction.
\end{abstract}

2000 Mathematics Subject Classification. 17B60.

Let $J=J(N, 1)$ be a quadratic Jordan algebra with 1 over a field $\Phi$ constructed as in [3] from an admissible nondegenerate cubic form $N$ with base point 1 . Let

$$
E=\left\{\left[\begin{array}{ll}
\alpha & a \\
b & \beta
\end{array}\right] \mid \alpha, \beta \in \Phi, a, b \in J\right\}
$$

Then it is shown in [1] that $E$ is a ternary algebra. Moreover, it is shown in [1] that $S=S(E, R(E))=E \oplus \tilde{E} \oplus \Phi u \oplus \Phi \tilde{u} \oplus \Phi U \oplus D$ is a Lie algebra of dimension 248, where $\tilde{E}$ denotes a second copy of $E$. (Recall from [1] that $D$ is the algebra of derivations of $E$ and $R(E)=\Phi U \oplus D$, the span of $\{R(x, y) \mid x, y \in E\} \cup\{U\}$. For $R=E \oplus \Phi u$, $U(E)=\left\{A \in \operatorname{Hom}_{\Phi}(R, R) \mid u A \in \Phi u\right.$ and $\left.E A \subseteq E\right\}$ is a Lie algebra with $[A B]=A B-B A$. Here $U \in U(E)$ such that $u U=2 u$ and $x U=x$, for all $x \in E$. So, $U$ is in the center of $U(E)$. Also $R(x, y) \in U(E)$, for $x, y \in E$, is defined by $u R(x, y)=\langle x, y\rangle u, z R(x, y)=$ $\langle z, x, y\rangle$, for all $z \in E$, where $\langle$,$\rangle is the bilinear form and \langle,$,$\rangle is the ternary form$ defining a ternary $\Phi$-module, as in [1].)

On the other hand, let $C$ be the algebra of split octonions and $J$ an arbitrary Jordan algebra of degree 3. Then according to Tits in [4], $L=\operatorname{Der}(C) \oplus C_{0} \otimes J_{0} \oplus \operatorname{Der}(J)$ is a Lie algebra, where $\operatorname{Der}(C)$ is the algebra of derivations of $C$ and $C_{0}$ is the subspace of $C$ whose elements have trace 0 .

THEOREM 1. There is an explicit isomorphism between the Lie algebras constructed by Tits and the Faulkner processes.

The proof of this theorem is the goal of this paper and the proof completes this paper.

Our attempt is to construct an isomorphism $\Phi: L \rightarrow S$ by breaking $\Phi$ into three parts $\Phi_{1}$ restricted to $\operatorname{Der}(C), \Phi_{2}$ restricted to $C_{0} \otimes J_{0}$, and $\Phi_{3}$ restricted to $\operatorname{Der}(J)$.

First we construct a candidate $S_{1}$ for the image of $\Phi_{1}$ in $S$ which in turn plays a significant role throughout the paper. This $S_{1}$ is obtained by replacing $J$ by $\Phi_{1}$ in the 
construction of $S$. Therefore,

$$
S_{1}=E_{1} \oplus \tilde{E}_{1} \oplus \Phi u \oplus \Phi \tilde{u} \oplus \Phi U \oplus D_{1}
$$

LEMMA 2. The dimension of $D_{1}=3$.

The study of the derivation algebra $D$ gives detailed information about $S$. So, we need to look at the action of $R\left(x_{1}, y_{1}\right), x_{1}, y_{1} \in E$, on an arbitrary element

$$
z=\left[\begin{array}{ll}
\alpha & a \\
b & \beta
\end{array}\right] \in E
$$

Let

$$
x_{i}=\left[\begin{array}{cc}
\alpha_{i} & a_{i} \\
b_{i} & \beta_{i}
\end{array}\right] \in E \quad \text { for } i=1,2
$$

Recall from [1] that $R(x, y)$ is the close derivation of $E$ if and only if $\rho(x, y)=$ $\langle x, y\rangle=0$. Then it is straightforward to check that $R\left(x_{12}, 1_{22}\right), R\left(x_{21}, 1_{11}\right)$, and $R\left(x_{12}, 1_{21}\right)$ are the only distinct derivations of $E$, where $x_{12}$ is the $2 \times 2$ matrix with $(1,2)$ th element equal to $x \in J_{0}$ and 0 elsewhere. In case $J=\Phi 1$, similar calculations and tables show that $R\left(1_{11}, 1_{21}\right), R\left(1_{22}, 1_{12}\right)$, and $R(1,1)$, where 1 is the $2 \times 2$ unit matrix in the last term, form a basis of $D_{1}$. Thus, Lemma 2 is proved.

Now it is clear that $\operatorname{Dim}\left(S_{1}\right)=14$ which is the same as $\operatorname{Dim}(\operatorname{Der}(C))$ as in [4]. So, $\Phi_{1}$ could be defined conveniently. Moreover, it is necessary to test that the role of $S_{1}$ in $S$ is the same as the role of $\operatorname{Der}(C)$ in $L$. As a matter of fact, this test will provide us with an idea for the definition of $\Phi_{2}$.

Now let $\left\{1_{11}, 1_{12}, 1_{21}, 1_{22}, \tilde{1}_{11}, \tilde{1}_{12}, \tilde{1}_{21}, \tilde{1}_{22}, u, \tilde{u}, U, R\left(1_{11}, 1_{21}\right), R\left(1_{22}, 1_{12}\right), R(1,1)\right\}$ be a basis of $S_{1}$. There are 7 copies of $J_{0}$ in $S$. Two copies in $E$, two in $\tilde{E}$ and three in $D$. Let these copies of $J_{0}$ be represented by $x_{12}, x_{21}, \tilde{x}_{12}, \tilde{x}_{21}, R\left(x_{12}, 1_{22}\right), R\left(x_{21}, 1_{11}\right)$, and $R\left(x_{12}, 1_{21}\right)$.

LEMmA 3. Let $x \in J_{0}$, then the $\Phi$-vector space spanned by the seven copies of $x$ is an $S_{1}$-module.

Proof. It is enough to show that the Lie product of the seven copies of $J_{0}$ with each of the generators of $S_{1}$ are elements of $J_{0}$. The computations are lengthy but straightforward. Only two parts are shown below and the rest are similar. The Lie product rule in [1, equation (2.8)] is used.

(1) $\left[x_{12}, 1_{11}\right]=\left[x_{12}, 1_{12}\right]=\left[x_{12}, 1_{21}\right]=\left[x_{12}, 1_{22}\right]=0,\left[x_{12}, \tilde{1}_{11}\right]=0,\left[x_{12}, \tilde{1}_{12}\right]=$ $R\left(x_{21}, 1_{11}\right),\left[x_{12}, \tilde{1}_{21}\right]=R\left(x_{12}, 1_{21}\right),\left[x_{12}, \tilde{1}_{22}\right]=\left[x_{12}, 1_{22}\right],\left[x_{12}, u\right]=0,\left[x_{12}, \tilde{u}\right]=\tilde{x}_{12}$, $\left[x_{12}, U\right]=x_{12},\left[x_{12}, R\left(1_{11}, 1_{21}\right)\right]=0,\left[x_{12}, R\left(1_{22}, 1_{12}\right)\right]=-\tilde{x}_{21},\left[x_{12}, R(1,1)\right]=x_{12}$.

(2) $\left[R\left(x_{12}, 1_{21}\right), 1_{11}\right]=\left[R\left(x_{12}, 1_{21}\right), 1_{22}\right]=0,\left[R\left(x_{12}, 1_{21}\right), 1_{12}\right]=2 x_{12},\left[R\left(x_{12}, 1_{21}\right), 1_{21}\right]$ $=2 x_{21},\left[R\left(x_{12}, 1_{21}\right), \tilde{1}_{11}\right]=\left[R\left(x_{12}, 1_{21}\right), \tilde{1}_{22}\right]=0,\left[R\left(x_{12}, 1_{21}\right), \tilde{1}_{12}\right]=2 \tilde{x}_{12},\left[R\left(x_{12}, 1_{21}\right)\right.$, $\left.\tilde{1}_{21}\right]=2 \tilde{x}_{21},\left[R\left(x_{12}, 1_{21}\right), u\right]=\left[R\left(x_{12}, 1_{21}\right), \tilde{u}\right]=\left[R\left(x_{12}, 1_{21}\right), U\right]=0,\left[R\left(x_{12}, 1_{21}\right)\right.$, $\left.R\left(1_{11}, 1_{21}\right)\right]=-2 R\left(x_{21}, 1_{11}\right),\left[R\left(x_{12}, 1_{21}\right), R\left(1_{22}, 1_{12}\right)\right]=2 R\left(x_{12}, 1_{22}\right),\left[R\left(x_{12}, 1_{21}\right)\right.$, $R(1,1)]=0$. 
Using the Lie product rule in the Faulkner's construction in [1], we have for $x, y \in J_{0}$, $\left[x_{12}, y_{12}\right]=\left\langle x_{12}, y_{12}\right\rangle u=0$. Similarly, $\left[x_{21}, y_{21}\right]=\left[\tilde{x}_{12}, \tilde{y}_{12}\right]=\left[\tilde{x}_{21}, \tilde{y}_{21}\right]=0$. Let

$$
z=\left[\begin{array}{cc}
\alpha & a \\
b & \beta
\end{array}\right] \in E, \quad \bar{O}=\left[\begin{array}{ll}
0 & 0 \\
0 & 0
\end{array}\right], \quad \bar{A}=\left[\begin{array}{ll}
0 & a \\
b & 0
\end{array}\right] .
$$

It is easy to check that $z\left[R\left(x_{12}, 1_{22}\right), R\left(y_{12}, 1_{22}\right)\right]=z\left[R\left(x_{21}, 1_{11}\right), R\left(y_{21}, 1_{11}\right)\right]=\bar{O}$ and $z\left[R\left(x_{12}, 1_{21}\right), R\left(y_{12}, 1_{21}\right)\right]=4 \bar{A}\left[R_{x}, R_{y}\right]$. The use of the ternary product $\langle,$,$\rangle as in [1],$ equations (1.6) and (2.3), may be needed. For future reference, we note that using similar computations implies $\left[R\left(x_{12}, 1_{21}\right), R\left(y_{12}, 1_{21}\right)\right]=R\left(x_{12}, y_{21}\right)-R\left(x y_{12}, 1_{21}\right)$.

As in [2, page 105], let $\{e 1, e 2, f 1, f 2, f 3, g 1, g 2, g 3\}$ be a basis of $C$. Then $C_{0}$ is spanned by $\{e 2-e 1, f 1, f 2, f 3, g 1, g 2, g 3\}$. Moreover, $\operatorname{Der}(C)$ is 14-dimensional, and is spanned by $\left\{\mathrm{D}_{f 1, f 2}, \mathrm{D}_{f 1, f 3}, \mathrm{D}_{f 2, f 3}, \mathrm{D}_{g 1, g 2}, \mathrm{D}_{g 1, g 3}, \mathrm{D}_{g 2, g 3}, \mathrm{D}_{e 1, g i}, \mathrm{D}_{e 2, f i}, \quad(i=1,2,3)\right.$, $\left.\mathrm{D}_{f 1, g 1},\left(\mathrm{D}_{f 2, g 2}-\mathrm{D}_{f 3, g 3}\right)\right\}$ from [4, page 77]. [Recall that $\mathrm{D}_{x, z}=\left[R_{x}, R_{y}\right]+\left[L_{x}, R_{z}\right]+$ [ $\left.L_{x}, L_{z}\right]$, where $R_{x}, L_{x}$ are the right and left multiplications]. But $C_{0}$ is 7-dimensional tells us that $L$ contains 7 copies of $J_{0}$. We name them, for $x \in J_{0}$, as $(e 2-e 1) \otimes x$, $f 1 \otimes x, f 2 \otimes x, f 3 \otimes x, g 1 \otimes x, g 2 \otimes x, g 3 \otimes x$. Note that $C_{0} \otimes J_{0}$ is a $\operatorname{Der}(C)$-module, because, for $a \in C_{0}, x \in J_{0},[a \otimes x, \mathrm{D}]=a \mathrm{D} \otimes x \in C_{0} \otimes J_{0}$, where $\mathrm{D} \in \operatorname{Der}(C)$. Moreover, for $x, y \in J_{0},[(e 2-e 1) \otimes x,(e 2-e 1) \otimes y]=\left[R_{x}, R_{y}\right] \in \operatorname{Der}(J)$, and $[f i \otimes x, f i \otimes y]=$ $[g i \otimes x, g i \otimes y]=0, i=1,2,3$.

Thus, comparing the above two paragraphs, we conclude that $(e 2-e 1) \otimes x$ and $R\left(x_{12}, 1_{21}\right)$ have similar roles in $L$ and $S$, respectively. Moreover, the annihilator of $(e 2-e 1) \otimes x$ is spanned by $\left\{\mathrm{D}_{f 1, g 1},\left(\mathrm{D}_{f 2, g 2}-\mathrm{D}_{f 3, g 3}\right), \mathrm{D}_{f 1, g 2}, \mathrm{D}_{f 2, g 3}, \mathrm{D}_{f 3, g 1}, \mathrm{D}_{f 1, g 3}, \mathrm{D}_{f 2, g 1}\right.$, $\left.\mathrm{D}_{f 3, g 2}\right\}$. The annihilator of $R\left(x_{12}, 1_{21}\right)$ is spanned by $\left\{1_{11}, 1_{22}, \tilde{1}_{11}, \tilde{1}_{22}, u, \tilde{u}, U, R(1,1)\right\}$ which can be seen in the proof of Lemma 3. Studying the actions of the annihilators of $R\left(x_{12}, 1_{21}\right)$ and $(e 2-e 1) \otimes x$ on the rest of the six copies of $J_{0}$, respectively, we define $\phi_{1}$ and $\phi_{2}$ in the following way: $\mathrm{D}_{f 1, g 2} \phi_{1}=-31_{11}, \mathrm{D}_{f 3, g 1} \phi_{1}=31_{22}, \mathrm{D}_{f 2, g_{3}} \phi_{1}=$ $3 \tilde{u}, \mathrm{D}_{f 1, g} \phi_{1}=3 \tilde{1}_{11}, \mathrm{D}_{f 2, g 1} \phi_{1}=3 \tilde{1}_{22}, \mathrm{D}_{f 3, g 2} \phi_{1}=-3 u, \mathrm{D}_{f 1, g 1} \phi_{1}=R(1,1),\left(\mathrm{D}_{f 2, g 2}-\right.$ $\left.\mathrm{D}_{f 3, g 3}\right) \phi_{1}=-3 U, \mathrm{D}_{e 2, g 1} \phi_{1}=R\left(1_{22}, 1_{12}\right), \mathrm{D}_{e 2, g 2} \phi_{1}=-1_{12}, \mathrm{D}_{e 2, g 3} \phi_{1}=\tilde{1}_{12}, \mathrm{D}_{e 1, f 1} \phi_{1}=$ $R\left(1_{11}, 1_{21}\right), \mathrm{D}_{e 1, f 2} \phi_{1}=\tilde{1}_{21}, \mathrm{D}_{e 1, f 3} \phi_{1}=1_{21}$, and $\left(f_{1} \otimes x\right) \phi_{2}=R\left((1 / 2) x_{21}, 1_{11}\right),\left(f_{2} \otimes\right.$ $x) \phi_{2}=(1 / 2) \tilde{x}_{21},\left(f_{3} \otimes x\right) \phi_{2}=(1 / 2) x_{21},\left(g_{1} \otimes x\right) \phi_{2}=R\left((1 / 2) x_{12}, 1_{22}\right),\left(g_{2} \otimes x\right) \phi_{2}=$ $-(1 / 2) x_{12},\left(g_{3} \otimes x\right) \phi_{2}=(1 / 2) \tilde{x}_{12}$. Recall from [4] that $[a \otimes x, \mathrm{D}]=a \mathrm{D} \otimes x$, for all $\mathrm{D} \in \operatorname{Der}(C), a \in C_{0}, x \in J_{0}$.

LEMMA 4. The mapping $\phi_{1}$ is an isomorphism and $\phi_{2}$ is a bijection.

Proof. The map $\phi_{1}$ is injective: let $\mathrm{D}_{1} \phi_{1}=\mathrm{D}_{2} \phi_{1}$ for $\mathrm{D}_{1}, \mathrm{D}_{2} \in \operatorname{Der}(C)$ and $\mathrm{D} 1 \neq \mathrm{D} 2$. We can write $\mathrm{D}_{1} \phi_{1}=\left(c_{1} \mathrm{D}_{f 1, g 2}+c_{2} \mathrm{D}_{f 3, g 1}+\cdots+c_{14} \mathrm{D}_{e 1, f 3}\right) \phi_{1}=c_{1}\left(-31_{11}\right)+c_{2}\left(31_{22}\right)+$ $\cdots+c_{14} 1_{21}=S_{1}$. Similarly, $\mathrm{D}_{2} \phi_{1}=k_{1}\left(-31_{11}\right)+k_{2}\left(31_{22}\right)+\cdots+k_{14} 1_{21}=S_{2}, c_{i}, k_{i} \in \Phi$ and $\phi_{1}$ is clearly linear. But $S_{1}=S_{2}$ implies $c_{i}=k_{i}$ and hence $\mathrm{D}_{1}=\mathrm{D}_{2}$.

The map $\phi_{1}$ is surjective: let $S \in S_{1}$. Then $S=c_{1}\left(-31_{11}\right)+c_{2}\left(31_{22}\right)+\cdots+c_{14} 1_{21}=$ $\left(c_{1} \mathrm{D}_{f 1, g 2}+c_{2} \mathrm{D}_{f 3, g 1}+\cdots+c_{14} \mathrm{D}_{e 1, f 3}\right) \phi_{1}=\mathrm{D}_{\phi_{1}}$ (say). But $\mathrm{D} \in \operatorname{Der}(C)$.

The map $\phi_{1}$ preserves Lie product: let $\mathrm{D}_{1}, \mathrm{D}_{2} \in \operatorname{Der}(C)$. To show that $\left[\mathrm{D}_{1} \phi_{1}, \mathrm{D}_{2} \phi_{1}\right]$ $=\left[\mathrm{D}_{1}, \mathrm{D}_{2}\right] \phi_{1}$. First we notice that for $a \otimes x \in C_{0} \otimes J_{0}$ and $\mathrm{D} \in \operatorname{Der}(C),\left[(a \otimes x) \phi_{2}, \mathrm{D} \phi_{1}\right]$ $=[a \otimes x, \mathrm{D}] \phi_{2}=(a \mathrm{D} \otimes x) \phi_{2}$ from the study of correspondences between $\operatorname{Der}(C)$ and $S_{1}$, and $\operatorname{Der}(C)$-module in $L$ and $S_{1}$-module in $S$ we discussed before. Using the Jacobi identity, we have, $\left[\left[a \otimes x, \mathrm{D}_{1}\right], \mathrm{D}_{2}\right]+\left[\left[\mathrm{D}_{1}, \mathrm{D}_{2}\right], a \otimes x\right]+\left[\left[\mathrm{D}_{2}, a \otimes x\right], \mathrm{D}_{1}\right]=0$. Thus we 
obtain $a\left[\mathrm{D}_{1}, \mathrm{D}_{2}\right] \otimes x=a \mathrm{D}_{1} \mathrm{D}_{2} \otimes x-a \mathrm{D}_{2} \mathrm{D}_{1} \otimes x$. Therefore, $\left[(a \otimes x) \phi_{2},\left[\mathrm{D}_{1} \phi_{1}, \mathrm{D}_{2} \phi_{1}\right]\right]=$ $\left[\left[(a \otimes x) \phi_{2}, \mathrm{D}_{1} \phi_{1}\right], \mathrm{D}_{2} \phi_{1}\right]-\left[\left[(a \otimes x) \phi_{2}, \mathrm{D}_{2} \phi_{1}\right], \mathrm{D}_{1} \phi_{1}\right]=\left(a \mathrm{D}_{1} \mathrm{D}_{2} \otimes x-a \mathrm{D}_{2} \mathrm{D}_{1} \otimes x\right) \phi_{2}$ $=\left(a\left[\mathrm{D}_{1}, \mathrm{D}_{2}\right] \otimes x\right) \phi_{2}=\left[a \otimes x,\left[\mathrm{D}_{1}, \mathrm{D}_{2}\right]\right] \phi_{2}$. Thus, $\left[(a \otimes x) \phi_{2},\left[\mathrm{D}_{1} \phi_{1}, \mathrm{D}_{2} \phi_{1}\right]\right]=[(a \otimes$ $\left.x) \phi_{2},\left[\mathrm{D}_{1}, \mathrm{D}_{2}\right] \phi_{1}\right]$. But, $\phi_{2}$ is bijective in the same way as $\phi_{1}$ is and by dimension count. Hence, $\phi_{1}$ preserves Lie product and $\phi_{1}$ is an isomorphism.

LEMMA 5. The centralizer of $S_{1}$ is $\left\{R \in \operatorname{Der}(E) \mid E_{1} R=0\right\}$.

Proof. Let the centralizer of $S_{1}$ be denoted by cent $\left(S_{1}\right)$. Then $\operatorname{cent}\left(S_{1}\right)=\{\mathrm{S} \in S \mid$ $\left.\left[\mathrm{S}, S_{1}\right]=0\right\}$. Let $\mathrm{S}=y+\tilde{e}+\alpha u+\beta \tilde{u}+R$ and $\mathrm{S}_{1}=y_{1}+\tilde{e}_{1}+\alpha_{1} u+\beta_{1} \tilde{u}+R_{1}$. Then, $\left[\mathrm{S}, y_{1}\right]=$ 0 implies $\left\langle y, y_{1}\right\rangle u-R\left(y_{1}, e\right)+\beta y_{1}-y_{1} R=0$. Hence, $\left\langle y, y_{1}\right\rangle=0, y_{1} R=-R\left(y_{1}, e\right)$, $\beta=0$. Similarly, $\left[\mathrm{S}, \tilde{e}_{1}\right]=0$ implies $\alpha=0,[\mathrm{~S}, u]=0$ implies $e=0, \rho(R)=0,[\mathrm{~S}, \tilde{u}]=0$ implies $\tilde{y}=0$ and finally, $\left[\mathrm{S}, R_{1}\right]=0$ implies $\left[R, R_{1}\right]=0$. Moreover, $\tilde{y}=0$ implies $y=0, e=0$ implies $\tilde{e}=0, y_{1} R=0$ implies $E_{1} R=0$. Hence $\mathrm{S}=R$ and $R$ is a derivation $E$. Thus, cent $\left(S_{1}\right)=\left\{R \in \operatorname{Der}(E) \mid E_{1} R=0\right.$ and $\left.\left[R, R_{1}\right]=0\right\}$. If $R_{1}=R_{1}\left(x_{1}, y_{1}\right)$ for $x_{1}, y_{1} \in E_{1}$ then $\left[R, R_{1}\left(x_{1}, y_{1}\right)\right]=-\left[R_{1}\left(x_{1}, y_{1}\right), R\right]=R_{1}\left(x_{1} R, y_{1}\right)+R_{1}\left(x_{1}, y_{1} R\right)=0$ for $E_{1} R=0$. Hence, $\operatorname{cent}\left(S_{1}\right)=\left\{R \in \operatorname{Der}(E) \mid E_{1} R=0\right\}$.

EXAMPLE 6. The bracket $\left[R\left(x_{12}, 1_{21}\right), R\left(y_{12}, 1_{21}\right)\right]$ is an element of cent $\left(S_{1}\right)$. This is clear from the computations after the proof of Lemma 3. For $z$ and $\bar{A}$ as defined in these computations, $z\left[R\left((1 / 2) x_{12}, 1_{21}\right), R\left((1 / 2) y_{12}, 1_{21}\right)\right]=\bar{A}\left[R_{x}, R_{y}\right], x, y \in J_{0}$. But, $\left[R_{x}, R_{y}\right] \in \operatorname{Der}(J)$, as we have seen before. It is an easy exercise that for $x, y \in J$ there exist $x 0, y 0 \in J_{0}$ such that $\left[R_{x}, R_{y}\right]=\left[R_{x 0}, R_{y 0}\right]$. Hence, for $x, y \in J_{0},\left[R_{x}, R_{y}\right]$ span $\operatorname{Der}(J)$. Write $\left[R_{x}, R_{y}\right]=D_{x, y}$. Then we define $\phi_{3}$ as follows: $\phi_{3}: \operatorname{Der}(J) \rightarrow$ $\operatorname{cent}\left(S_{1}\right)$ by $\left(D_{x, y}\right) \phi_{3}=\left[R\left((1 / 2) x_{12}, 1_{21}\right), R\left((1 / 2) y_{12}, 1_{21}\right)\right]$. (Recall from [4] that for a central simple Jordan algebra $J$ of degree 3 there is a linear function $T($ ) such that $x=(1 / 3) T(x)+x 0, x \in J, x 0 \in J_{0} . T(1)=3 \cdot x * y=x \cdot y-(1 / 3) T(x \cdot y) 1$, for $x, y \in J_{0}$.)

\section{LEMMA 7. The map $\phi_{3}$ is an isomorphism.}

Proof. The map $\phi_{3}$ is injective: let $\left(\mathrm{D}_{x, y}\right) \phi_{3}=0$. Then clearly, $\mathrm{D}_{x, y}=0$ from the above discussions.

The map $\phi_{3}$ is surjective: recall that $S=E \oplus \tilde{E} \oplus \Phi u \oplus \Phi \tilde{u} \oplus \Phi U \oplus D$, where $D=$ $\left\{\sum_{i} R\left(x_{i}, y_{i}\right) \mid \sum_{i}\left\langle x_{i}, y_{i}\right\rangle=0\right\}$ as in [1, Section 4].

We have $S_{1}=E_{1} \oplus \tilde{E}_{1} \oplus \Phi u \oplus \Phi \tilde{u} \oplus \Phi U \oplus D_{1}$. Moreover, $J=J_{0} \oplus \Phi 1$, as in [4]. These show that we write $S$ as a direct sum of $S_{1}$, the vector space generated by the copies of $J_{0}$ and some derivations in $D$. In fact we will show that $S=S_{1} \oplus \operatorname{Im}\left(\phi_{2}\right) \oplus \operatorname{Im}\left(\phi_{3}\right)$. So, it is enough to show that each element of $D$ can be written as a direct sum of elements in these three parts. For each $i$, let

$$
R\left(x_{i}, y_{i}\right)=R\left(\left[\begin{array}{cc}
1 & x \\
x^{\prime} & 1
\end{array}\right],\left[\begin{array}{cc}
1 & y \\
y^{\prime} & 1
\end{array}\right]\right), \quad x, x^{\prime}, y, y^{\prime} \in J
$$

Using linearity, $R\left(x_{i}, y_{i}\right)=R\left(1_{11}, 1_{11}\right)+R\left(1_{11}, y_{12}\right)+R\left(1_{11}, y_{21}^{\prime}\right)+R\left(1_{11}, 1_{22}\right)+$ $R\left(x_{12}, 1_{11}\right)+R\left(x_{12}, y_{12}\right)+R\left(x_{12}, y_{21}^{\prime}\right)+R\left(x_{12}, 1_{22}\right)+R\left(x_{21}^{\prime}, 1_{11}\right)+R\left(x_{21}^{\prime}, y_{12}\right)+$ $R\left(x_{21}^{\prime}, y_{21}^{\prime}\right)+R\left(x_{21}^{\prime}, 1_{22}\right)+R\left(1_{22}, 1_{11}\right)+R\left(1_{22}, y_{12}\right)+R\left(1_{22}, y_{21}^{\prime}\right)+R\left(1_{22}, 1_{22}\right)$. Using 
$y=(1 / 3) T(y) 1+y_{0}, y_{0} \in J_{0}$, we get $R\left(1_{11}, y_{12}\right)=(1 / 3) T(y) R\left(1_{11}, 1_{12}\right)+R\left(1_{11}\right.$, $\left.\left(y_{0}\right)_{12}\right)$. Similarly, $R\left(1_{12}, y_{12}^{\prime}\right)$ and $R\left(x_{12}, y_{12}\right)$ are sum of elements in $S_{1}$ and elements in the copies of $J_{0}$. Using a remark after Lemma 3 , we have $R\left(\left(x_{0}\right)_{12},\left(y_{0}\right)_{21}\right)=$ $\left[R\left(\left(x_{0}\right)_{12}, 1_{21}\right), R\left(\left(y_{0}\right)_{12}, 1_{21}\right)\right]+R\left(\left(x_{0} y_{0}\right)_{12}, 1_{21}\right)$. Thus, $R\left(x_{12}, y_{21}\right)$ is a sum of elements in $S_{1}$, in $\operatorname{Im}\left(\phi_{2}\right)$, and in $\operatorname{Im}\left(\phi_{3}\right)$. Moreover, $R\left(x_{12}, y_{21}\right)-R\left(y_{21}, x_{12}\right)=$ $\left\langle x_{12}, y_{21}\right\rangle U$, by [1, equation (2.5)]. Hence, we have shown that $S=S_{1} \oplus \operatorname{Im}\left(\phi_{2}\right) \oplus \operatorname{Im}\left(\phi_{3}\right)$. Since $\phi_{3}$ is injective, we obtain $=S_{1} \oplus \operatorname{Im}\left(\phi_{2}\right) \oplus \operatorname{cent}\left(S_{1}\right)$. Thus, $\operatorname{cent}\left(S_{1}\right)=\operatorname{Im}\left(\phi_{3}\right)$ and $\phi_{3}$ is surjective.

The map $\phi_{3}$ preserves Lie product: let $A=\left[R\left((1 / 2) x_{12}, 1_{21}\right), R\left((1 / 2) y_{12}, 1_{21}\right)\right]$ and $B=\left[R\left((1 / 2) x_{12}^{\prime}, 1_{21}\right), R\left((1 / 2) y_{12}^{\prime}, 1_{21}\right)\right]$ for $x, y, x^{\prime}, y^{\prime} \in J_{0}$. Then $z[A, B]=z A B-$ $z B A=\bar{A}\left[\mathrm{D}_{x, y}, \mathrm{D}_{x^{\prime}, y^{\prime}}\right]$. By definition, $\left[\mathrm{D}_{x, y}, \mathrm{D}_{x^{\prime}, y^{\prime}}\right] \phi_{3}=[A, B]=\left[\left(\mathrm{D}_{x, y}\right) \phi_{3},\left(\mathrm{D}_{x^{\prime}, y^{\prime}}\right) \phi_{3}\right]$.

REMARK 8. The Lie product of an element in $\operatorname{cent}\left(S_{1}\right)$ with an element in a copy of $J_{0}$ gives an element in a copy of $J_{0}$.

This shows that the behavior of $\operatorname{cent}\left(S_{1}\right)$ in $S$ is the same as that of $\operatorname{Der}(J)$ in $L$.

To complete the proof of the Theorem the only thing left is to check that $\phi_{3}$ defined on $C_{0} \otimes J_{0}$ preserves Lie product. From [4, page 22], for $x, y \in J_{0}$ we have [ $f 1 \otimes x, g 2 \otimes$ $y]=(1 / 12)\langle x, y\rangle \mathrm{D}_{f 1, g 2}+f 1 * g 2 \otimes x * y-(f 1, g 2)\left[R_{x}, R_{y}\right]=(1 / 12) T(x y) \mathrm{D}_{f 1, g 2}+$ $(f 1 g 2-(1 / 2) T(f 1 g 2)) \otimes x * y+(1 / 2) T(f 1 g 2)\left[R_{x}, R_{y}\right]=(1 / 12) T(x y) \mathrm{D}_{f 1, g 2}$. (Note that thus $f 1 g 2=0,(f 1, g 2)=(1 / 2) T(f 1, \tilde{g} 2)=(1 / 2) T(f 1,-g 2)=-(1 / 2) T(f 1 g 2)$. Therefore, $[f 1 \otimes x, g 2 \otimes y] \phi_{3}=(1 / 12) T(x y)\left(-31_{11}\right)$. On the other hand, for

$$
H=\left[\begin{array}{cc}
-\frac{1}{4} T(x, y) & 0 \\
0 & 0
\end{array}\right],
$$

$\left[\left(f_{1} \otimes x\right) \phi_{3},(g 2 \otimes y) \phi_{3}\right]=\left[R\left((1 / 2) x_{12}, 1_{11}\right),-(1 / 2) y_{12}\right]=(1 / 2) y_{12} R\left((1 / 2) x_{12}, 1_{11}\right)=$ $H=(1 / 12) T(x y)\left(-31_{11}\right)$, where $T(x, y)=T(x y)$. Similarly, $[f 1 \otimes x, g 3 \otimes y] \phi_{3}=$ $(1 / 12) T(x y)\left(3 \tilde{1}_{11}\right)=\left[(f 1 \otimes x) \phi_{3},(g 3 \otimes y) \phi_{3}\right]$. Also $[f 2 \otimes x, g 1 \otimes y] \phi_{3}=(1 / 12) \times$ $T(x y)\left(3 \tilde{1}_{22}\right)=\left[(f 2 \otimes x) \phi_{3},(g 1 \otimes y) \phi_{3}\right]$. And $[f 2 \otimes x, g 3 \otimes y] \phi_{3}=(1 / 12) T(x y)(3 \tilde{u})=$ $\left[(f 2 \otimes x) \phi_{3},(g 3 \otimes y) \phi_{3}\right]$. Similarly, one can continue to check for $[f 3 \otimes x, g 1 \otimes y]$ and $[f 3 \otimes x, g 2 \otimes y]$. And $[f 1 \otimes x, f 2 \otimes y] \phi_{3}=\left(-(1 / 12) T(x y) \mathrm{D}_{e 2, g 3}+g 3 \otimes x * y\right) \phi_{3}=$ $(1 / 4)(x \times y) \tilde{1}_{12}=\left[(f 1 \otimes x) \phi_{3},(f 2 \otimes y) \phi_{3}\right]$, where $x \times y=2 x y-T(x y)$. Similar computations for $[f 1 \otimes x, f 3 \otimes y],[g 1 \otimes x, g 2 \otimes y]$, and $[g 1 \otimes x, g 3 \otimes y]$. We also have, $[f 2 \otimes x, f 3 \otimes y] \phi_{3}=-(1 / 12) T(x y) R\left(1_{22}, 1_{12}\right)+R\left((1 / 2)(x * y)_{12}, 1_{22}\right)$. For

$$
z=\left[\begin{array}{ll}
\alpha & a \\
b & \beta
\end{array}\right] \in E, \quad K=\frac{1}{4}\left[\begin{array}{cc}
0 & \alpha x \times y \\
a \times(x \times y) & T(b, x \times y)
\end{array}\right],
$$

$z\left([f 2 \otimes x, f 3 \otimes y] \phi_{3}\right)=K=z\left(\left[(f 2 \otimes x) \phi_{3},(f 3 \otimes y) \phi_{3}\right]\right)$. Similarly, $[g 2 \otimes x, g 3 \otimes$ $y] \phi_{3}=\left((1 / 12) T(x y) \mathrm{D}_{f 1, g 1}+(1 / 2)(e 2-e 1) \otimes x * y+(1 / 2) \mathrm{D}_{y, x}\right) \phi_{3}=(1 / 12) T(x y)$ $R(1,1)-(1 / 12) T(x y) R\left(1_{12}, 1_{21}\right)+(1 / 4) R\left(y_{12}, x_{21}\right)$. Then for

$$
L=\frac{1}{4}\left[\begin{array}{cc}
\alpha T(x y) & a T(x y)-\{a x y\} \\
-b T(x y)+\{b y x\} & -\beta T(x y)
\end{array}\right],
$$


$z\left([f 1 \otimes x, g 1 \otimes y] \phi_{3}\right)=L=z\left[(f 1 \otimes x) \phi_{3},(g 1 \otimes y) \phi_{3}\right]$. Similar computations are for $[f 3 \otimes x, g 3 \otimes y]$. We also have, $[f 2 \otimes x, g 2 \otimes y] \phi_{3}=(1 / 4) R\left(y_{12}, x_{21}\right)=[(f 2 \otimes$ $\left.x) \phi_{3},(g 2 \otimes y) \phi_{3}\right]$. To check the above two equations, one may need that $\left(\mathrm{D}_{f 2, g 2}\right) \phi_{3}=$ $\left[\mathrm{D}_{e 1, f 2}, \mathrm{D}_{e 2, g 2}\right] \phi_{3}=\left[\tilde{1}_{21},-1_{12}\right]=R\left(1_{12}, 1_{21}\right)$. Similarly, $\left(\mathrm{D}_{f 3, g 3}\right) \phi_{3}=R\left(1_{21}, 1_{12}\right)$. Since $[(e 2-e 1) \otimes x, f 1 \otimes y] \phi_{3}=\left(-(1 / 6) T(x y) \mathrm{D}_{e 1, f 1}-f 1 \otimes x * y\right) \phi_{3}=-(1 / 2) R\left((x y)_{21}, 1_{11}\right)$. One could use that $\mathrm{D}_{e 2-e 1, f 1}=-2 \mathrm{D}_{e 1, f 1}$. For

$$
N=\frac{1}{2}\left[\begin{array}{cc}
T(a, x y) & b \times x y \\
\beta x y & 0
\end{array}\right],
$$

$z\left([(e 2-e 1) \otimes x, f 1 \otimes y] \phi_{3}\right)=N=z\left[((e 2-e 1) \otimes x) \phi_{3},(f 1 \otimes y) \phi_{3}\right]$. Similar computations are for $[(e 2-e 1) \otimes x, g 1 \otimes y]$. We also have, $[(e 2-e 1) \otimes x, f 2 \otimes y] \phi_{3}=$ $-(1 / 2) x y \tilde{1}_{21}=\left[((e 2-e 1) \otimes x) \phi_{3},(f 2 \otimes y) \phi_{3}\right]$. Similar computations are for [ $(e 2-$ $e 1) \otimes x, f 3 \otimes y],[(e 2-e 1) \otimes x, g 2 \otimes y]$, and $[(e 2-e 1) \otimes x, g 3 \otimes y]$.

Thus, we have shown that $\phi_{3}$ preserves Lie product. Hence $\phi$ is an isomorphism and the proof of the theorem is completed.

ACKNOWLEDGEMENT. This paper constitutes a part of the author's Master's thesis written under the direction of Professor Michael L. Racine at the University of Ottawa, Ottawa, Ontario, Canada. It is dedicated to the author's parents.

\section{REFERENCES}

[1] J. R. Faulkner, A construction of Lie algebras from a class of ternary algebras, Trans. Amer. Math. Soc. 155 (1971), 397-408. MR 45\#3494. Zbl 215.38503.

[2] J. E. Humphreys, Introduction to Lie Algebras and Representation Theory, Graduate Texts in Mathematics, vol. 9, Springer-Verlag, New York, 1978. MR 81b:17007. Zbl 447.17001.

[3] K. McCrimmon, The Freudenthal-Springer-Tits constructions of exceptional Jordan algebras, Trans. Amer. Math. Soc. 139 (1969), 495-510. MR 39\#276. Zbl 175.02703.

[4] R. D. Schafer, An Introduction to Nonassociative Algebras, Pure and Applied Mathematics, vol. 22, Academic Press, New York, 1966. MR 35\#1643. Zbl 145.25601.

SUDHIR R. NATH: 13200 ISANTI STREET, BLAINE, MN 55449, USA

E-mail address: sudhir.r.nath@we11sfargo.com 


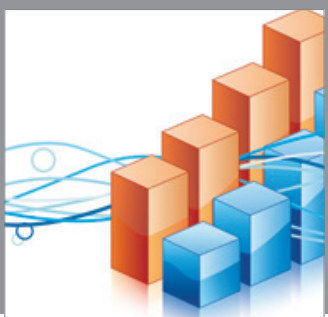

Advances in

Operations Research

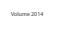

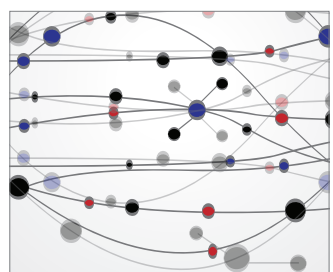

\section{The Scientific} World Journal
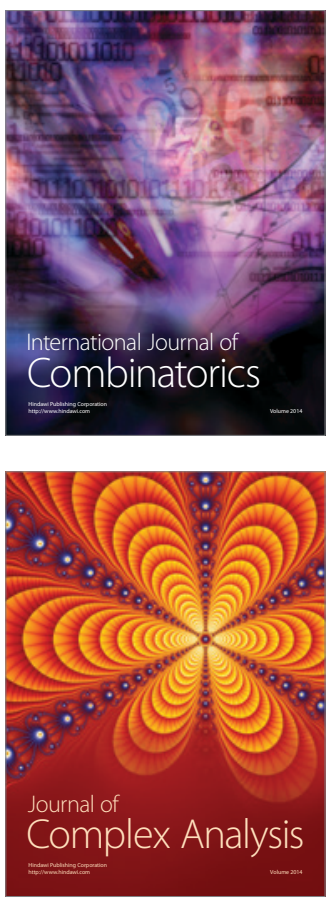

International Journal of

Mathematics and

Mathematical

Sciences
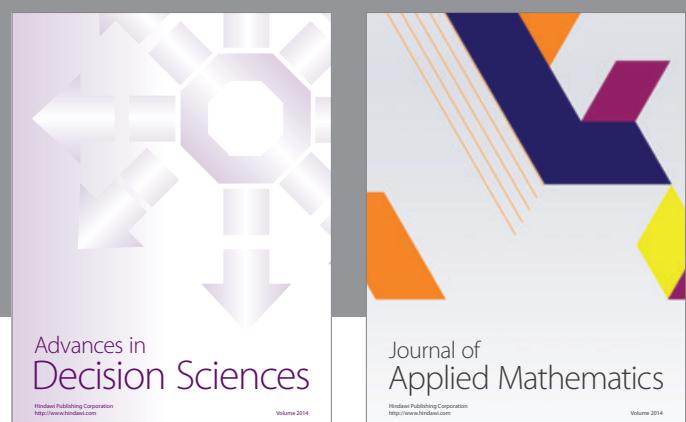

Journal of

Applied Mathematics
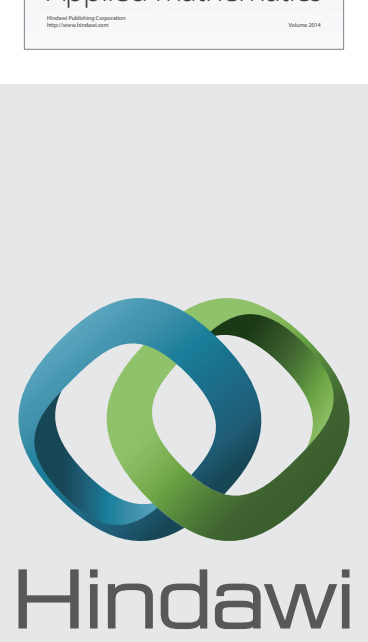

Submit your manuscripts at http://www.hindawi.com
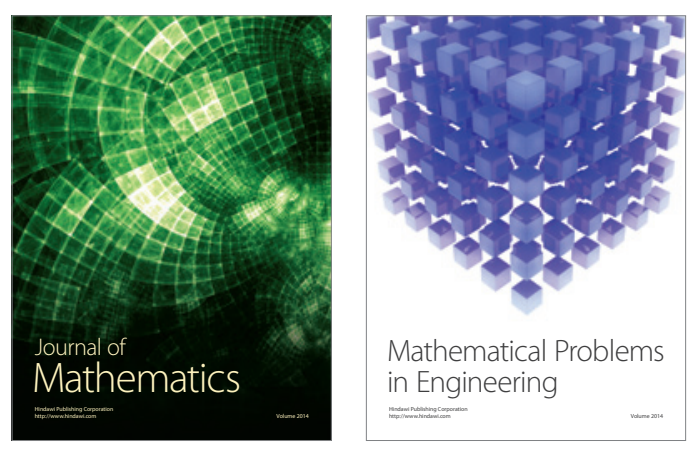

Mathematical Problems in Engineering
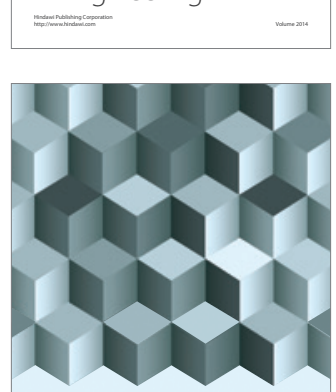

Journal of

Function Spaces
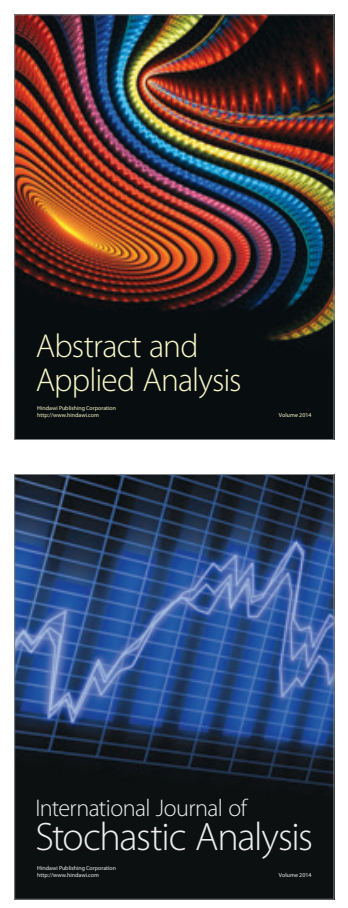

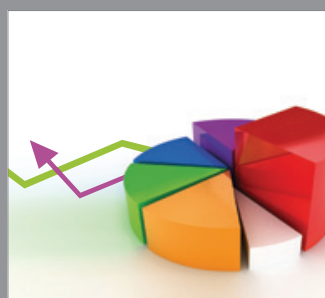

ournal of

Probability and Statistics

Promensencen
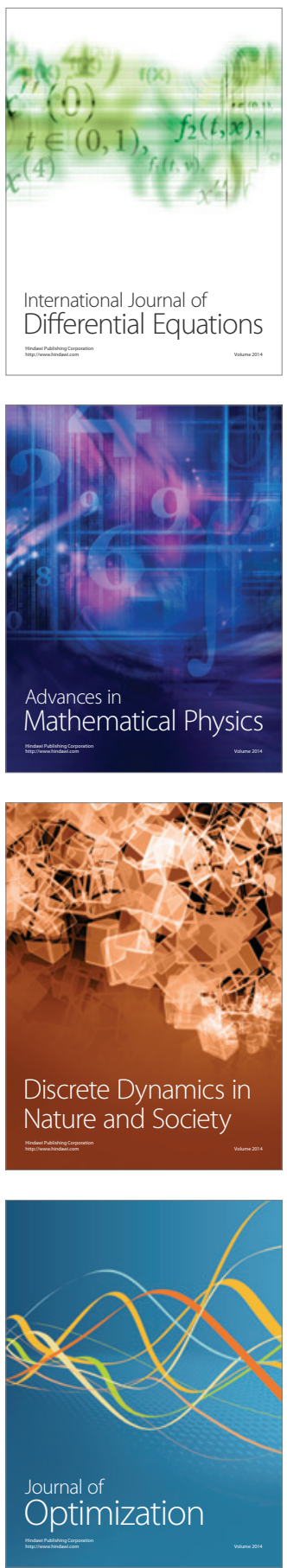